University of Nebraska - Lincoln

DigitalCommons@University of Nebraska - Lincoln

Congreso internacional sobre iconografía

precolombina, Barcelona 2019. Actas.

8-20-2020

\title{
Oficiantes Mochica Medio en San José de Moro: El Sacerdote Lechuza y La Sacerdotisa
}

Karim Ruiz Rosell

Follow this and additional works at: https://digitalcommons.unl.edu/actas2019

Part of the American Material Culture Commons, Indigenous Studies Commons, Museum Studies Commons, Other History of Art, Architecture, and Archaeology Commons, and the Other Languages, Societies, and Cultures Commons

This Article is brought to you for free and open access by the Zea E-Books at DigitalCommons@University of Nebraska - Lincoln. It has been accepted for inclusion in Congreso internacional sobre iconografía precolombina, Barcelona 2019. Actas. by an authorized administrator of DigitalCommons@University of Nebraska - Lincoln. 


\section{Oficiantes Mochica Medio en San José de Moro: El Sacerdote Lechuza y La Sacerdotisa}

\author{
Dr. Karim Ruiz Rosell \\ Profesor de la Universidad San Ignacio de Loyola (Lima, Perú). \\ Correo: karimruizrosell@gmail.com
}

\begin{abstract}
Resumen
Las tumbas Mochica Medio de El Sacerdote Lechuza y La Sacerdotisa de San José de Moro (SJM) esbozan una identidad individual particular y relacionan a los individuos enterrados con una élite ceremonial en la que habrían desempeñado funciones de oficiantes adscritas a los rituales en los que dichos personajes aparecen en la iconografía. Por un lado, la tumba del Sacerdote Lechuza, llena de alusiones iconográficas a la lechuza (metales y cerámica) que remiten a la figura del Sacerdote Lechuza; por otro lado, la tumba de la Sacerdotisa, con varios elementos del "Tema de la Presentación" tallados en concha que ejercen obligatoriamente de antecedente a la tradición de Sacerdotisas enterradas en SJM en periodos posteriores.
\end{abstract}

Palabras clave: Mochica Medio, Funerario, Oficiantes, Sacerdotisa, Lechuza

\begin{abstract}
The Middle Moche tombs of The Barn Owl Priest and The Priestess found in San Jose de Moro (SJM) outline a particular individual identity and link the buried individuals with a ceremonial elite in which they would have performed functions of officiants attached to the rituals in which these characters appear in the iconography. On one hand, the tomb of the Barn Owl Priest, full of iconographic allusions to the barn owl (metals and pottery) that refer to the figure of the Barn Owl Priest; on the other hand, the tomb of the Priestess, with several elements from the "Presentation Theme" carved in shell necessarily represent as a predecessor to the tradition of Priestesses buried in SJM in later periods.
\end{abstract}

Keywords: Middle Moche, Funerary, Officiants, Priestess, Barn Owl

\section{(2) \\ шшшшسше \\ Tradiciones funerarias en el Valle de Jequetepeque y San José de Moro}

Desde que en 1992 se descubrieran las tumbas de las Sacerdotisas en SJM, fue evidente la relevancia de este centro ceremonial y cementerio de la cultura Mochica; la profusión de tumbas y la riqueza de los objetos hallados en ellas confirmaba la presencia de una élite que hacía uso del lugar para llevar a cabo sus rituales religiosos, así como el entierro de sus miembros más importantes. No obstante, las prácticas funerarias de élite parecía que se habían empezado a producir durante el Periodo Mochica Tardío (y posteriores), mientras que durante el periodo inmediatamente precedente, el Periodo Mochica Medio, todavía se trataba de una sociedad sin demasiadas diferencias sociales (por lo menos reflejadas en sus prácticas funerarias); es cierto que existían algunos ejemplos de tumbas con algunos objetos excepcionales pero, aparentemente, asociados a sus habilidades artesanales, no a su estatus social (Castillo y Rengifo, 2008).

De lo que no se tenía constancia era de la presencia de una élite explícitamente ligada a las prácticas religiosas o funerarias, lo cual no dejaba de sorprender dadas las características del propio sitio arqueológico. Fue tras las excavaciones realizadas a partir de 2005 en el Área 38 de SJM que se empezó a vislumbrar la presencia de

Publicado en Congreso internacional sobre iconografía precolombina, Barcelona 2019. Actas, Victòria Solanilla Demestre, editora (Lincoln, Nebraska: Zea Books, 2020). https://doi.org/10.32873/unl.dc.zea.1254 
una élite sacerdotal durante el Periodo Mochica Medio. Esta élite sacerdotal no sólo se habría enterrado con ricos objetos que los diferenciaban del resto de individuos que se enterraban en este cementerio, sino también con algunos ricos objetos símbolo de su desempeño sacerdotal en vida, marcando de esta forma su pertenencia a la élite pero, al mismo tiempo, proyectando su identidad asociada al sacerdocio y, multiplicando así, los elementos de distinción jerárquica (Ruiz, 2013).

Es importante destacar la continuidad tanto de la presencia arqueológica en general como de la presencia de elementos más específicos que corroboran la existencia de una élite sacerdotal en SJM desde la ocupación correspondiente al Periodo Mochica Medio hasta el Periodo Transicional. El contexto de ofrendas de llamas que se encontró en la zona evidencia la importancia del sector en el que se encontraron estas tumbas en épocas posteriores, estableciendo una cierta relación de sacralidad entre la ubicación inicial de las tumbas Mochica Medio y los eventos llevados a cabo en periodos posteriores (Goepfert, 2006; Ruiz, 2013).

Además de la existencia de este peculiar contexto con ofrendas de cientos de llamas, se han realizado hallazgos pertenecientes a periodos posteriores que confirman la pervivencia de un carácter sacro-funerario de mucha importancia en esa área del cementerio además de los propios contextos. En primer lugar, la existencia de muros perimetrales que delimitaban áreas donde ubicar grandes cámaras funerarias durante los Periodos Mochica Tardío y Transicional (Castillo y Rengifo, 2008); en segundo lugar, la cercanía con la Huaca Choddoff y lo que conlleva la ubicación de las tumbas cerca de los monumentos sagrados; y, finalmente, los "hallazgos extraoficiales" fuera del perímetro arqueológico (confirmando la presencia de tumbas Mochica Medio de gran riqueza). Todos estos factores hacen prever la existencia de contextos con las mismas características o, aunque distintas, que ayuden a corroborar la existencia de una élite sacerdotal en SJM desde el Periodo Mochica Medio.

Lo primero que debemos destacar para entender la importancia de estas tumbas es su singularidad, en el sentido más amplio de la palabra. Si, por un lado, son tumbas excepcionales por su forma, tamaño y contenido, por otro lado, son las únicas que se han encontrado en SJM con estas características, lo cual nos deja poco margen para contrastar y corroborar los hallazgos. No hay que olvidar que la extensión real del cementerio es mucho mayor que la actual zona de excavación, extendiéndose hacia las áreas que actualmente se encuentran bajo las casas de los pobladores. Precisamente, esta situación ya ha reportado durante años los ya mencionados "hallazgos extraoficiales" por parte de los habitantes del actual pueblo de SJM, los cuales corroboran nuestra teoría de un núcleo de tumbas de élite Mochica Medio y nos ayudan a contrastar el material encontrado; lamentablemente, el análisis descontextualizado de estas ofrendas no nos permite confirmar muchos de los aspectos que tratamos de demostrar.

Otro aspecto que se ha podido constatar con estos nuevos hallazgos es la estrecha relación que existió entre las élites de SJM y las de Pacatnamú. Es cierto que esta relación se intuía tanto por la proximidad geográfica y las relaciones político-militares dentro del valle de Jequetepeque (Castillo et al, 2008), como por algunos elementos puntuales encontrados en tumbas de ambos cementerios (Del Carpio, 2008); pero un paralelismo tan directo entre sus patrones funerarios (morfología, características de las ofrendas e iconografía representada) no se había podido establecer hasta ahora.

Esta constatación ha reforzado la idea de que en el valle de Jequetepeque existieron estrechos lazos entre los habitantes de los distintos asentamientos; si se tenía constancia de los lazos políticos (gestión y control de los canales) y sociales (patrones de asentamiento), ahora quedaba claro que compartían también lazos religiosos, por lo menos por lo que respecta a sus prácticas funerarias. No olvidemos que SJM fue un centro religioso de peregrinación sin una población permanente que lo habitara; se podría decir que actuó como algunas ciudades griegas durante las guerras helénicas, que asumían el papel de ciudad neutral para que se pudieran realizar los rituales olímpicos. Los habitantes del valle de Jequetepeque habrían escogido este lugar como centro ritual y religioso para enterrar a sus congéneres, en especial aquellos que destacaron jerárquicamente dentro de la sociedad Mochica, y habrían viajado desde sus respectivas poblaciones (en algunos casos bastante alejadas) hasta este enclave para llevar a cabo dichas exequias.

Habiendo observado las características de las complejas y ricas tumbas de SJM y habiéndolas comparado con las también complejas y ricas tumbas excavadas en Pacatnamú (Donnan y Cock, 1986; Ubbelohde-Doering 1983), podemos afirmar que la élite del Valle de Jequetepeque escogió ambos lugares para el entierro habitual de sus miembros. Ahora bien, hoy en día todavía desconocemos cuáles pudieron haber sido los criterios de esta élite para escoger uno u otro cementerio durante el Periodo Mochica Medio. Es difícil aventurar una afirmación al res- 
pecto debido a los poco ejemplos con los que se cuenta hasta el momento, pero lo que sí se puede concluir es que SJM, al tener un carácter ceremonial además de funerario, albergó los entierros de las élites sacerdotales del valle; ni Pacatnamú ni ningún otro cementerio del valle de Jequetepeque cuentan con contextos de individuos enterrados con ajuares que claramente los ligaran a la élite religiosa Mochica Medio.

Curiosamente, el valle de Jequetepeque sí cuenta con ejemplos de este tipo de tumbas ligadas a una élite sacerdotal para el periodo Mochica Temprano en el sitio de Dos Cabezas (Donnan, 2008). Esto resulta intrigante, a la par que casi concluyente con respecto a la hipótesis que presentamos, en cuanto que ofrece un paréntesis temporal en el que se debería poder rastrear una tradición y patrones funerarios que, por el contrario, parecía que no hubiera existido. Es posible, entonces, que el cementerio de Pacatnamú sólo acogiera los entierros de sus élites gobernantes y demás personajes importantes por sus habilidades artesanas (Donnan y Cock, 1986) pero SJM, además, acogiera los entierros de los miembros de la élite sacerdotal que llevaban a cabo los rituales del sitio. Las diferencias genéticas que existieran entre personas que habitaran el mismo SJM y las que habitaran otros lugares del valle serían mínimas (imperceptibles, en verdad), por lo que es prácticamente imposible sustentar esta teoría; además, siempre podría existir la posibilidad de que los sacerdotes y sacerdotisas fueran traídos de otros lugares, incluso desde más allá del valle de Jequetepeque (aunque en este caso el análisis de ADN sí nos podría esclarecer algunas cuestiones).

A pesar de ello, no es alocado pensar que, tratándose como se trata de un centro ceremonial de gran tamaño y con una abundancia tan grande de tumbas, las exigencias rituales que demandaba SJM habrían planteado la necesidad de una presencia permanente de un "cuerpo" religioso; esto, sin embargo, contrasta con la baja presencia de estructuras consideradas permanentes en los estratos pertenecientes a este periodo. El único lugar donde esta élite sacerdotal habría podido habitar en SJM es en las huacas, las cuales han sido excavadas sólo parcialmente y, las que lo han sido, presentaron ocupaciones más tardías (Chimú) con áreas domésticas de ocupación permanente ${ }^{1}$; esta ocupación, aunque tardía, podría ser un indicador de la presencia de estas áreas de ocupación permanente en periodos como el Mochica Medio.
Los rituales de la muerte Mochicas estaban entre los más importantes dentro de esta cultura, siendo aquellos en los que invertían más riqueza material (ofrendas) y social (mano de obra) (Castillo, 2000). Ya hemos visto muchos ejemplos de la riqueza material y también hemos visto la fuerza de trabajo que se requería para la construcción de la estructura funeraria (que todavía debió ser más en el caso de las tumbas de cámara Tardías o Transicionales); pero a esta inversión de capital humano hay que sumarle toda la producción de comida y, especialmente, de bebida que debió acompañar dichos rituales (Castillo, ibidem).

Cuanto mayor debió ser la importancia del occiso, mayor debió ser el número de personas que asistieron y que prepararon todo el ritual; la posición social del individuo enterrado, por tanto, era directamente proporcional con la abundancia (en todos los sentidos) durante el ritual de entierro. Esta abundancia se reflejaba, no sólo en las ofrendas que se depositaban al interior de la tumba sino también en los sacrificios de animales que se hacían para los asistentes, en los litros de chicha que se preparaban para la celebración y en los rituales paralelos al entierro mismo, que podían incluir música, danzas, desfiles, etc. Al fin y al cabo, como dice Castillo (2000), "el papel fundamental de los rituales de élite era dar continuidad a la comunidad luego de la ruptura del orden que significaba la muerte de un individuo importante", por lo que todo el evento, en realidad, era un fenómeno social y no una simple "despedida" de carácter más privado.

\section{Las tumbas de los Oficiantes}

Pero vayamos a los elementos específicos que nos han hecho catalogar a estas tumbas como los lugares finales de reposo de las élites del Periodo Mochica Medio. Generalmente, en arqueología, no suele existir evidencia de las "acciones" llevadas a cabo por los individuos investigados y se tiende a interpretar esas "acciones" a partir de los "restos" que estas dejan. En el caso de la arqueología Mochica, que no cuenta con un registro directo escrito de su historia y costumbres, la interpretación de las "acciones" resulta todavía más compleja. Sin embargo, la riqueza decorativa del arte Mochica ha reportado tanto detalle descriptivo, e incluso narrativo, que ha dado pie a la interpretación minuciosa de sus hipotéticas "acciones".

1. Para el periodo Mochica Medio se encontraron ya en 2007 ocupaciones en la Huaca Chodoff, pero eran contextos de carácter doméstico y funerario de baja complejidad. 
Los dos casos presentados en este artículo ejemplifican estos dos tipos de "restos" que nos ayudan a interpretar cómo fue su entorno y cómo se desarrolló la sociedad Mochica en alguna de las facetas de su vida. Por un lado, tenemos el ejemplo de la representación de la lechuza en varios soportes y formas, todo ello producto de la observación de la naturaleza que los rodeaba y su posterior síntesis y divinización. Por otro lado, tenemos el ejemplo de la síntesis de la representación de una escena a sus tres elementos más significativos (sacerdotisa con copa, guerrero y panoplia), lo cual resulta en un ejercicio de abstracción de las "acciones" que los rodeaban. Si en el primer caso estaríamos hablando de una hipotética comparación entre el arte descriptivo Mochica y la "fotografía", en el segundo caso se podría comparar con el "video", puesto que nos narra una acción dinámica y no un mero elemento estático de su entorno.

El hecho de haber encontrado prácticas funerarias de élite Mochica Medio con tantas similitudes entre SJM y el cementerio de Pacatnamú, tanto en la morfología de las propias tumbas y el contenido de las mismas como en la iconografía asociada a las ofrendas halladas, nos lleva a pensar en una "identidad cultural" sobre una mera "identidad individual" (Castillo, 2005); es decir, que hubo una élite en el valle que estableció los patrones con los que debían ser enterrados, estipulando desde la forma de las tumbas hasta su contenido. Posiblemente, el proceso del propio ritual con el que estas élites se enterraron también estaba pautado pero, como venimos mencionando, las "acciones", en sí mismas, no nos heredan evidencia.

\section{El Sacerdote Lechuza}

Centrándonos en el primer caso, el de la tumba del $\mathrm{Sa}$ cerdote Lechuza, nos damos cuenta de que la suma de los elementos encontrados a modo de ofrenda (tanto en cantidad como en calidad), definen un rango elevado del individuo enterrado (Ver Fig.1). Ya hemos visto que los elementos que configuran tanto su ajuar como las ofrendas perfilan una "identidad cultural" (la idea del $\mathrm{Sa}$ cerdote Lechuza) pero también una "identidad personal" (las ofrendas al individuo). Como dice Castillo (2005), "las identidades y las posiciones sociales se recrearon en las tumba a través de la combinación de artefactos, del tratamiento de los cuerpos y de la construcción de las tumbas".
Esta identidad cultural se crea a partir de los elementos que hemos ido identificando de este contexto funerario. Algunas de estas ofrendas tendrían relación directa con la vida de este personaje (objetos usados en vida para desempeñar su papel de sacerdote), mientras que las otras tendrían relación con su muerte (objetos realizados exclusivamente para el sepelio $\mathrm{u}$ ofrendadas en ese momento); las representaciones de lechuzas en la cerámica, las representaciones de lechuzas asociadas al rostro de una divinidad en las sonajas y la propia presencia del collar sonaja formarían parte del primer grupo, mientras que todos los elementos de ajuar personal ${ }^{2}$ (pectoral, brazaletes, orejeras y tocado), los sacrificios de animales (llamas) y la propia morfología excepcional de la tumba formarían parte del segundo grupo.

Viendo con detalle alguna de estas ofrendas nos hemos percatado de esta suma de elementos para construir una identidad. Todo el conjunto de las 15 ofrendas cerámicas (Ver Fig. 2) que hemos analizado, por ejemplo, muestra por sí solo la relevancia e importancia que debió tener en vida el individuo; $y$, viendo más allá, nos acercan a la identidad del individuo que se enterró con ellas. Si bien es cierto que la cerámica, como objeto por sí solo, no siempre determina la importancia de un entierro (otro tipo de objetos y materiales resultan más diagnósticos), el número y las características de éstas ya definen un rango por encima de lo usual entre las prácticas funerarias Mochica Medio de SJM.

Pero lo que todavía resulta más significativo es la ofrenda de los "collares sonaja", no sólo por su mera presencia, sino también por las relaciones que establece con muchas representaciones en las que diversos personajes aparecen llevando estos "collares sonaja" (Ver Fig. 3). Tras haber analizado la pieza en sí, tanto en el conjunto como cada "disco" por separado, se puede afirmar que es un objeto de gran valor, con una fuerte carga artística y conceptual, que debió requerir de un esfuerzo de la comunidad (en material y mano de obra) y que, además, fue hecho y roto exclusivamente para funcionar como ofrenda al individuo de la tumba del Sacerdote Lechuza.

$\mathrm{Al}$ analizar las representaciones de lechuzas en el Periodo Mochica Medio, ya existe una distinción entre las representaciones naturalistas, sintéticas y antropomorfizadas. Esta distinción se manifiesta claramente en el caso de las sonajas puesto que se combina la forma de

2. En el caso del ajuar personal, estaría funcionando para ambas categorías, puesto que podrían ser tanto ofrendas funerarias como elementos de la propia liturgia con la que vinculamos al personaje. 

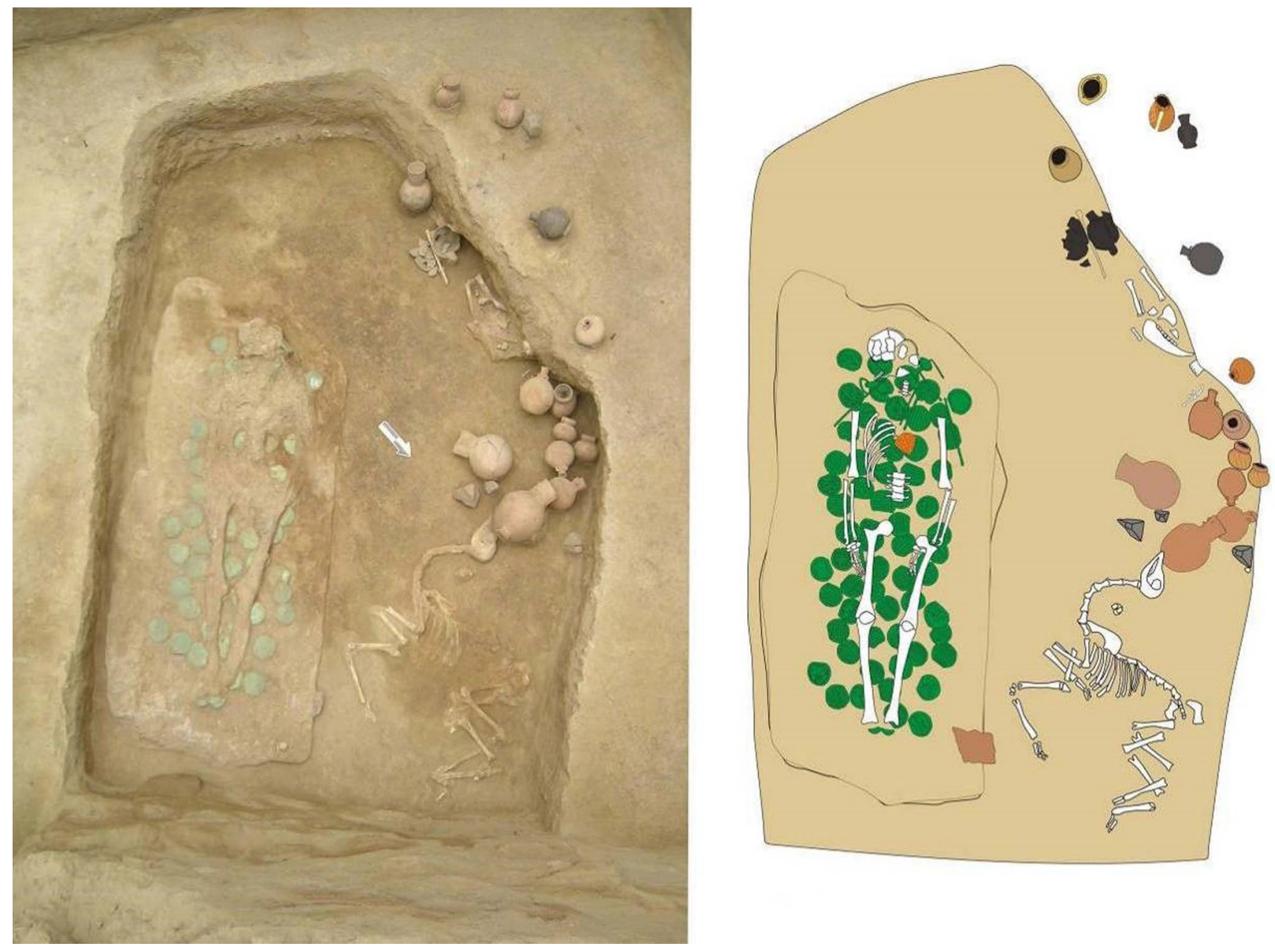

Figura 1: Dibujo de la tumba del Sacerdote Lechuza (PASJM).

la lechuza natural (morfológicamente identificada como lechuza de campanario), con la de la lechuza divinizada (antropomorfizada). También hemos visto que los motivos representados (lechuza y divinidad) nos remiten a la recreación de una identidad para el individuo enterrado, es decir, que hacen referencia a su vida o una parte de su vida: su papel en rituales llevados a cabo en SJM. Pero tanto los motivos individualmente como el objeto en conjunto nos remiten a varias representaciones de personajes en el arte Mochica en los que estos ejercen el papel de sacerdotes o divinidades (o individuos caracterizados como tales).

Una de estas representaciones era la del Sacerdote Lechuza, en el que, aunque hemos visto que está muy claro su rol en alguna de las escenas de la iconografía y su identificación se hace difícil en algunos casos, no dejan de ser evidentes los lazos que crean estas ofrendas con esta figura de la iconografía. Desde la obvia presencia de las representaciones de lechuzas (en cerámica y metales) hasta la ausencia misma de tocado en un contexto tan complejo, son elementos que denotan una clara intención de crear una identidad a partir de los objetos asociados.

También cabe mencionar el hecho de que este personaje con rasgos felinos aparezca asociado a otras ofrendas con representaciones de lechuzas simplificadas que se pueden confundir con búhos (confusión que se produce incluso en la propia naturaleza), lo cual nos podría llevar a pensar que el que está representado en las sonajas es el "Guerrero del Búho". Llegados a este punto, es interesante destacar el hecho de que Makowski (2000) puntualiza que el papel de esta divinidad en los rituales en los que participa es casi siempre pasivo, pero que delega sus funciones en una serie de "lugartenientes" que realizan funciones guerreras o sacerdotales. Estos personajes asumen las funciones de un oficiante para que 


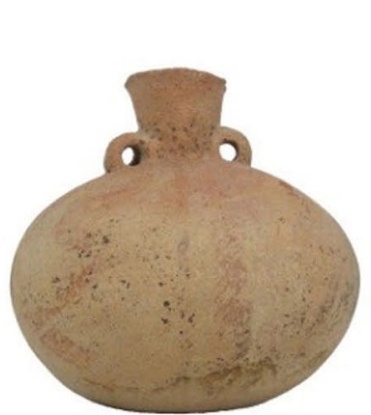

C1

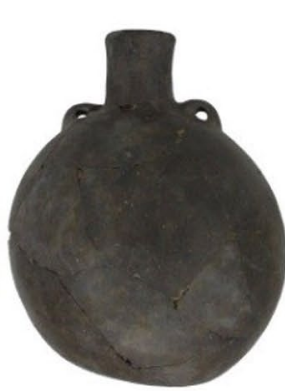

C2

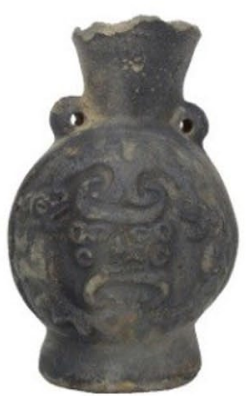

C3

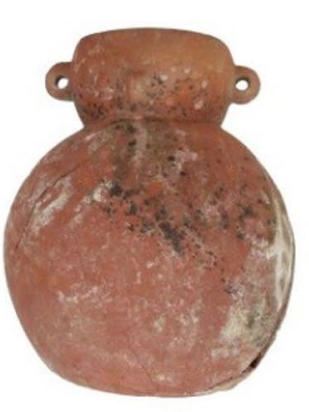

C4

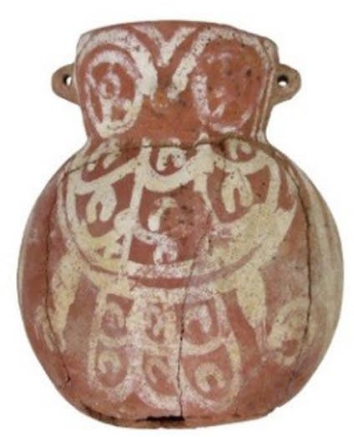

C5

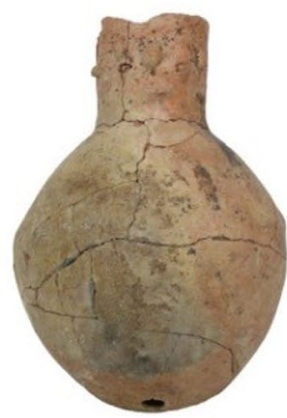

C6

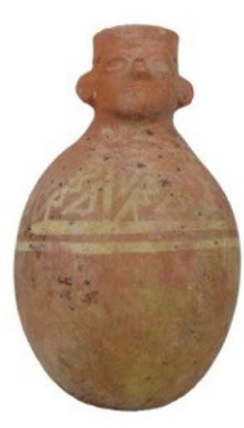

C7

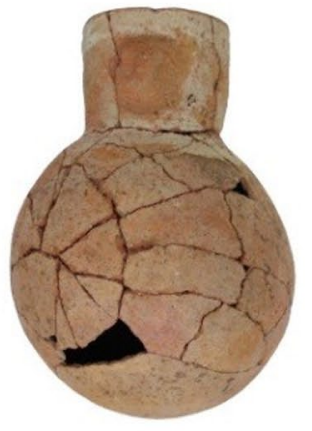

C8

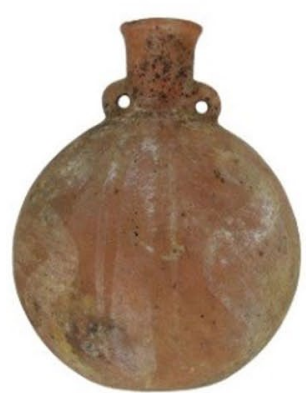

C9

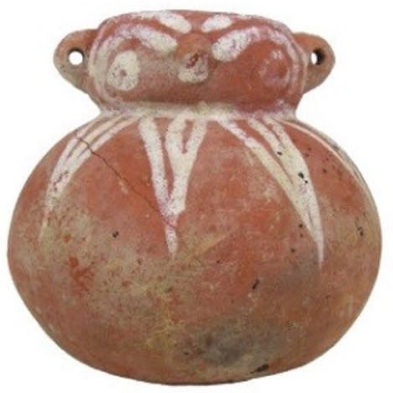

C10

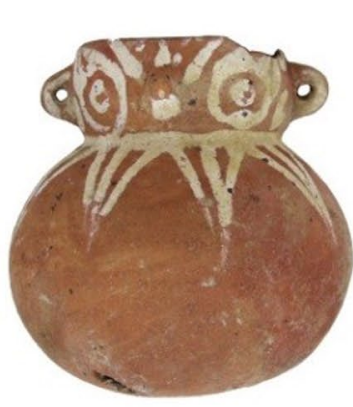

C11

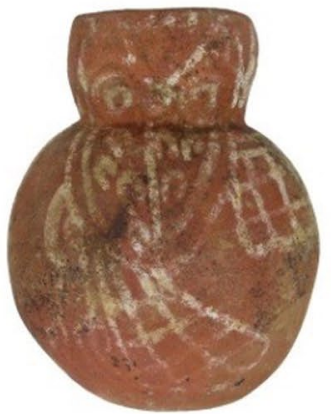

C12

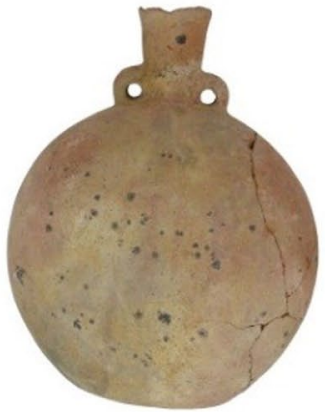

C13

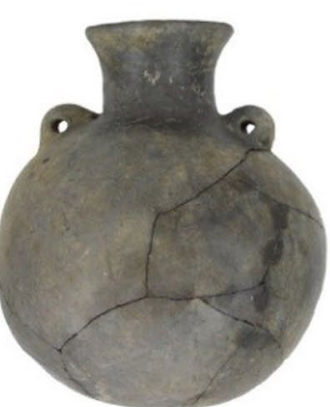

C14

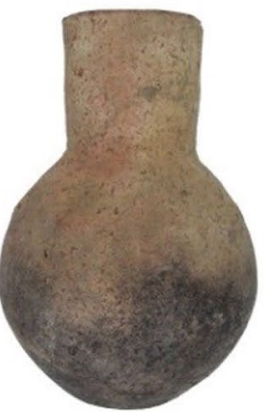

C15

Figura 2: Cerámica encontrada en la tumba del Sacerdote Lechuza (PASJM).

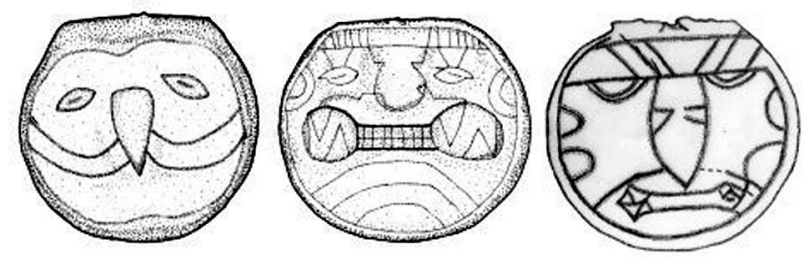

Figura 3: Discos 1, 7 y 35 de la tumba del Sacerdote Lechuza con representaciones de lechuzas, personaje sobrenatural y la síntesis de ambos (PASJM). 
el "Guerrero del Búho" presencie la ceremonia y reciba las ofrendas, pero al mismo tiempo adquieren algunos de los atributos y símbolos de la divinidad de la cual son oficiantes.

Ya fuera la representación de uno u otro personaje, lo que resulta evidente es que este collar sonaja con representaciones de lechuzas y divinidades era un objeto asociado a personajes importantes con un marcado rol litúrgico dentro de la iconografía Mochica (y, por lo tanto, reconocidos también como tales dentro de su sociedad). La presencia a modo de ofrenda de este objeto, además del hecho de que se inutilizara para que "muriera" con su dueño, enfatiza el carácter único del individuo enterrado, así como su relevancia dentro de la sociedad.

Por último, no olvidemos los sacrificios de llamas encontrados en este contexto funerario. Aunque era bastante habitual el sacrificio de llamas a modo de ofrenda en las tumbas Mochica Medio, generalmente, la parte ofrendada solía serla cabeza y las patas, es decir, las partes que en verdad no se podían consumir o eran de menor valor; sin embargo, lo que no era nada habitual era encontrar una llama completa sacrificada. Este último elemento también indicaba la excepcionalidad de dicho entierro confiriéndole más importancia al individuo enterrado.

Una vez caracterizado este Sacerdote Lechuza, resulta especialmente interesante la representación encontrada sobre una botella de asa estribo con decoración de línea fina, en la que aparece una escena encabezada por un personaje con rostro de lechuza (Ver Fig. 4); la presencia de este individuo con rostro de lechuza ya es interesante de por sí, pero más importante todavía es la presencia de los elementos dibujados que se asocian con ésta. En pri-

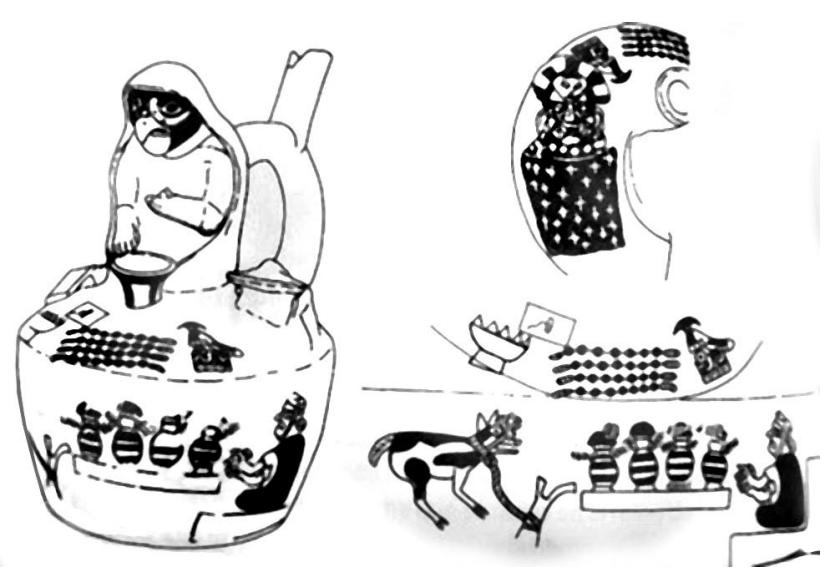

Figura 4: Dibujo de vasija con personaje ornitomorfo modelado y detalle de las ofrendas pintadas (Golte, 2009). mer lugar, vemos una serie de "collares-sonaja" (como los de la tumba del Sacerdote Lechuza) extendidos a modo de ofrenda; en segundo lugar, observamos una repisa con ofrendas de cerámica frente a un personaje sentado y una llama amarrada y lista para ser sacrificada (vasijas de cerámica y una llama eran otras ofrendas encontradas en la tumba del Sacerdote Lechuza). Finalmente, se observa lo que podría ser un fardo funerario con el cuerpo envuelto en un textil y el rostro de una divinidad con rasgos felinos y vistiendo un "collar sonaja". Toda esta escena bien podría haber representado un ritual en el que participara este Sacerdote Lechuza o, incluso, su propio ritual funerario.

\section{La Sacerdotisa}

Por lo que respecta al segundo caso, el de la tumba de la Sacerdotisa (Ver Fig.5), nos encontramos ante una situación todavía más interesante por lo que respecta al historial de investigación y descubrimientos de SJM, ya que los elementos descritos perfilan a la mujer enterrada como una oficiante asociada al "Tema de la Presentación”, que

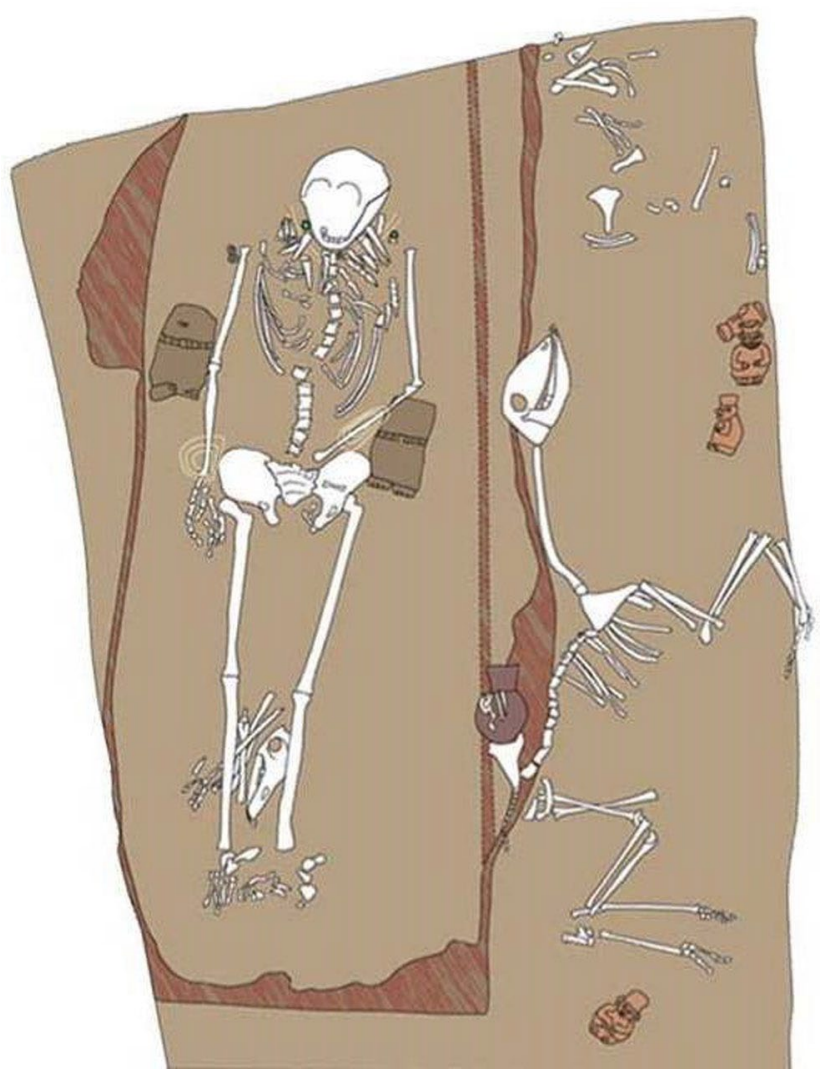

Figura 5: Dibujo de la tumba de la Sacerdotisa (PASJM). 
es, en parte, llevado a cabo por la Sacerdotisa o Mujer Sobrenatural (Donnan y McLelland, 1999) (Ver Fig. 6). El hecho de que se tratara de una mujer de la élite ceremonial ya es evidente por las características formales de la tumba en sí misma (en especial por las dimensiones), y se refuerza con la presencia de objetos como el pectoral, los collares y los brazaletes, así como por el mismo hecho de haber sido enterrada dentro de un ataúd.

De las asociaciones encontradas al interior del ataúd hay que destacar, la presencia de 2 figurinas de arcilla no cocida, endurecidas al sol, en las que se representan dos mujeres con tocados o cabezas bilobuladas que se sostienen las trenzas sobre el pecho. Se pudieron identificar algunos rasgos de dichas figuritas en el momento de excavarlas, pero el hecho de que no hubieran sido cocidas imposibilitó la preservación de las mismas; sin embargo, el azar hizo que en una de las tumbas aledañas (M-U1514) se encontrara una ofrenda de esas mismas figurinas pero esta vez de barro cocido (Ver Fig. 6).

Pero lo que definitivamente la asocia con una élite ceremonial, y en este caso con un personaje concreto de este ceremonial, es el hallazgo de su colgante de 8 piezas talladas en concha (Ver Fig. 7). Entre las representaciones de estas piezas cada una tiene tallado un diseño distinto: cuatro de ellas representan personajes antropomorfos con tocados, tres de ellas representan porras o panoplias y la última representa a un personaje, con tocado y pectoral, que está en actitud oferente sosteniendo una copa. Una de esas porras o panoplias habría ejercido de enganche para el resto, puesto que tenía un triple orificio por el que se habría introducido el cordel para atar al resto. La aparente individualidad de estas figuras talladas esconde, en realidad, una compleja narrativa que nos traslada a la conocida escena de la iconografía del Periodo Mochica Tardío del "Tema de la Presentación" (Ver Fig. 8).

Este "Tema de la Presentación" tallado en concha, presenta elementos que ejercen obligatoriamente de antecedente a la tradición de Sacerdotisas enterradas en SJM durante los Periodos Mochica Tardío y Transicional; por lo tanto, el vínculo se extiende al ceremonial llevado a cabo durante el Periodo Mochica Medio en zonas más al norte, como Sipán, y en zonas más al sur, como Huaca de la Luna. Como hemos ido viendo, la disparidad de tradiciones y estilos a lo largo del territorio Mochica no se correspondía con una aparente homogeneidad en las prácticas religiosas; es por este motivo que se encuentra rastros de las mismas prácticas religiosas (y los correspondientes reflejos en las manifestaciones artísticas de

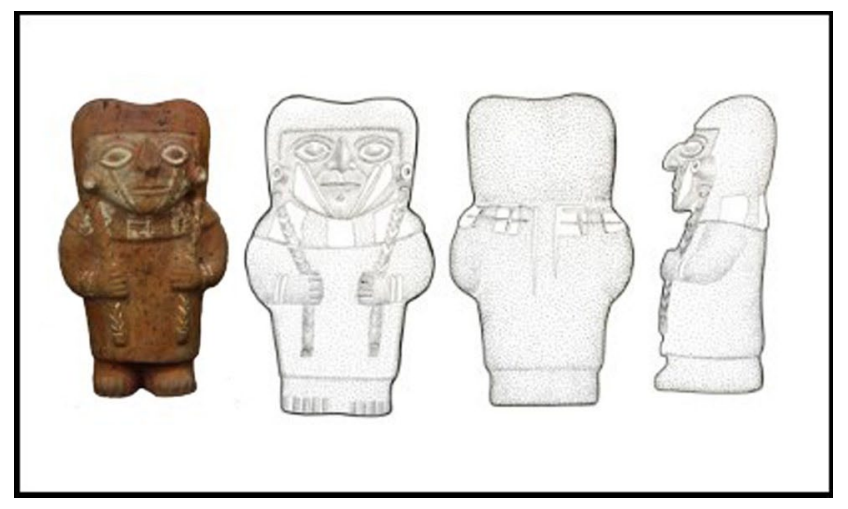

Figura 6: Fotografía y dibujos de figura escultórica hallada en una tumba asociada a la de la Sacerdotisa y que presenta las mismas características que las figuras no cocidas encontradas en ésta (PASJM).

estas) a lo largo de todo el territorio Mochica. Y esto no nos remite sólo a las prácticas funerarias asociadas al entierro de estas Sacerdotisas, sino que también nos remite al papel que debieron desempeñar durante su vida en los rituales del lugar. Por este motivo, podría ser considerada como una predecesora de la tradición de Sacerdotisas en SJM, es decir, una especie de "Proto-Sacerdotisa".

\section{Conclusiones}

Varios objetos asociados a estos personajes, además, suelen ser usados en rituales, como es el caso de la valva de spondylus y las sonajas de la tumba del Sacerdote Lechuza o de los amuletos colgantes de la tumba de esta Sacerdotisa. También hay otros elementos muy comunes en las escenas de ofrendas rituales que aparecen en la iconografía, como la llama o la cerámica, si bien en este caso parecen ser elementos más bien ligados al entierro del personaje en sí mismo y no a los rituales que habrían desempeñado en vida. Si a todos estos elementos sumamos los objetos del ajuar personal que acompañaron a los individuos enterrados (pectorales, orejeras, brazaletes, tocados, etc.) es inevitable aceptar la idea de que trataron de crear las identidades funerarias de personajes asociados al ceremonial.

Todos los elementos encontrados (ofendas y ajuar) junto a los individuos enterrados tienen un peso sustancial (tanto en cantidad como en calidad) en la conformación de un ritual funerario que los legitimaba como élite de su época. Lo que consolida esta teoría es la presencia de complejos objetos de cobre dorado (orejeras), de llamas sacrificadas, de parafernalia ritual (so- 


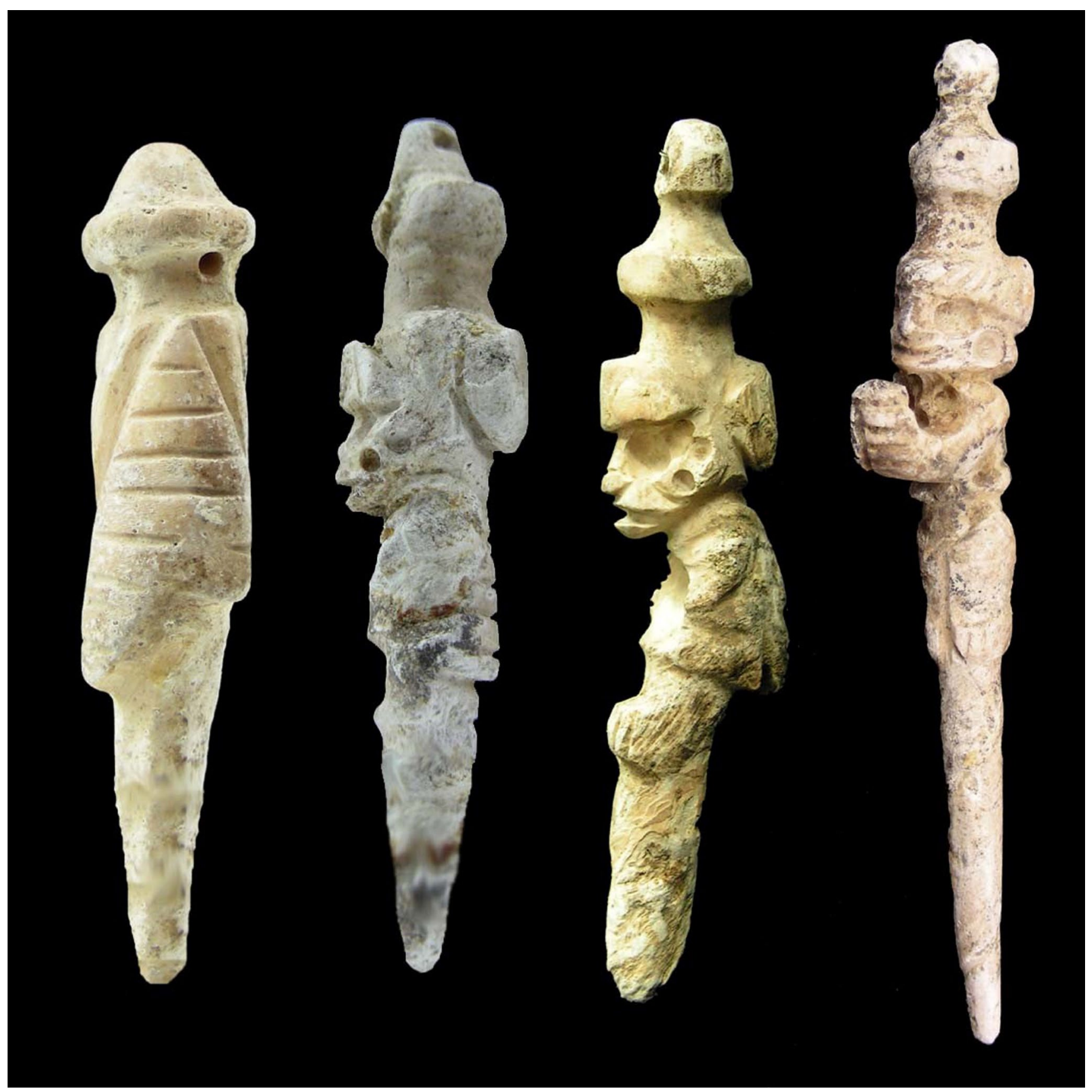

Figura 7: Detalle de 4 de los colgantes más representativos (panoplia, guerreros y sacerdotisa) pertenecientes al individuo de la tumba de la Sacerdotisa (PASJM).

najas, vasos y colgantes), de pectorales, de brazaletes y de collares (de cuentas); pero estos objetos son bastante variados y dispares entre sí, además de ser personalizados, lo que los puede alejar de una tradición funeraria homogénea.

Se podría decir que el común denominador entre estos contextos sea la presencia de los recipientes de cerá- mica con características que vinculan las tumbas de SJM entre sí y, a su vez, con algunas tumbas de Pacatnamú. Estos recipientes reflejan la mano de artistas que trabajaban bajo ciertos dictados temáticos y estilísticos, aunque los resultados fueran diversos como consecuencia de los distintos grados de habilidad de cada uno de ellos. El uso de los mismos recursos artísticos para producir el mismo 


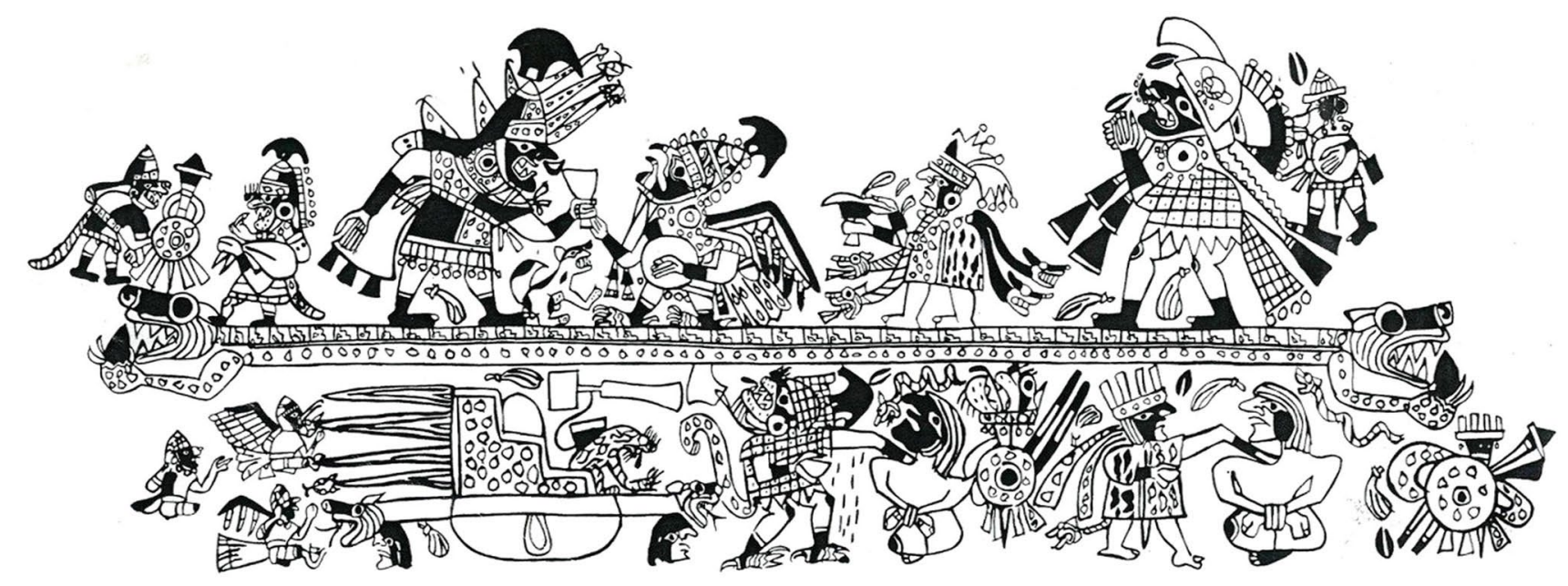

Figura 8: Decoración en línea fina con el Tema de la Presentación encontrado sobre una botella de asa estribo (Donnan y McClelland, 1999).

tipo de recipiente (forma y estilo) en distintas tumbas de SJM y Pacatnamú refuerza la teoría de una élite del valle que trataba de consolidar una misma tradición alrededor de sus rituales funerarios.

Es posible que siga existiendo la duda de si estas escenas que aparecen en el arte son realmente las representaciones de la vida de las divinidades o de los rituales recreados por seres humanos, pero es evidente que esos rituales sí existieron, como demuestra la presencia física de los objetos litúrgicos representados en el arte (en muchos casos con huellas de uso); en cambio, por decirlo de algún modo, no tenemos la certeza de que esas divinidades existieran. Por lo cual, tenderíamos a creer que las representaciones en el arte, especialmente las mencionadas escenas complejas representadas en "línea fina" (o sobre otros pocos soportes), se refieren a escenas interpretadas por seres humanos ataviados con elementos que los asemejaban al concepto que ellos mismos tenían de sus divinidades.

Hemos visto que los hallazgos arqueológicos que se han producido los últimos años a lo largo de toda la región Mochica, han reportado abundantes pruebas de que los objetos que se habían identificado en la iconografía eran objetos reales que eran usados en los rituales o, por lo menos, eran fabricados para ser ofrendados en los sepelios de las élites sacerdotales Mochica. La presencia en estos múltiples sitios de uno u otro objeto extienden el vínculo ceremonial en el uso de estos objetos desde zonas más al norte, como Sipán (Alva, 2004), hasta zonas más al sur, como Huaca de la Luna (Castillo y Uceda, 2008).
Como conclusión al análisis de estas tumbas, especialmente a la iconografía asociada a ellas, es necesario hacer hincapié en dos aspectos esenciales. En primer lugar, hay que resaltar los múltiples paralelismos que se han establecido entre estos contextos funerarios y algunos contextos funerarios de Pacatnamú y, en menor medida, de Huaca de la Luna y Sipán. En segundo lugar, hay que destacar las características específicas que presentan los personajes enterrados en estos contextos, ya que esbozan una identidad particular para cada uno de ellos y los relaciona con una élite ceremonial (ajuares y ofrendas) que habría existido durante el Periodo Mochica Medio en SJM.

Estos individuos tenían algún tipo de relación con Pacatnamú, ya fuera porque llegaron procedentes de este sitio, trayendo consigo su propia tradición funeraria, o bien porque, por algún motivo, les interesó vincularse a las tradiciones funerarias y a la élite allí imperantes. Tanto la morfología misma de las tumbas como la tipología y la simbología de muchas de sus asociaciones ejemplifican este vínculo. En especial, cabe destacar la similitud entre las ofrendas cerámicas de la tumba del Sacerdote Lechuza y la cerámica encontrada en las tumbas A1 y EI de Pacatnamú, así como la profusa presencia figuras de lechuzas en varios objetos de estas tumbas (Donnan y Cock, 1986).

Estaríamos hablando, entonces, de los primeros individuos directamente asociados al ceremonial de SJM para el Periodo Mochica Medio, puesto que para la ocupación funeraria durante otros periodos ha habido nuevos ha- 
llazgos desde que se descubriera la tumba de la primera Sacerdotisa en 1992. Hemos visto que existieron tumbas del Periodo Mochica Medio con personajes asociados a un ajuar por encima de la media, pero claramente no tenían relación con el ceremonial o no se ha podido definir, con total exactitud, si tuvieron un papel directo en las actividades rituales del cementerio de SJM.

Si bien es cierto que estas escenas mencionadas corresponden a la iconografía que encontramos eminentemente plasmada en objetos del Periodo Mochica Tardío (sobre todo en botellas decoradas con línea fina), no hay que olvidar que es muy probable que los rituales se hubieran llevado a cabo desde mucho antes, aunque no hubieran sido representados en la iconografía. Además, no hay que olvidar que la complejidad iconográfica fue mucho mayor durante el Mochica Tardío que durante el Mochica Medio, especialmente en la representación del ceremonial de la región Mochica Norte.

Todos estos aspectos, por lo tanto, confirman que nos encontramos ante las dos tumbas de élite más complejas para el Periodo Mochica Medio en SJM. Entonces, es lógico pensar que, probablemente, las tumbas Mochica Medio de élite de SJM se habrían ubicado en zonas distintas a las excavadas hasta el momento y que, tal vez, nos encontremos ante un clúster o núcleo funerario de tumbas de bota Mochica Medio que se articulen alrededor de un epicentro formado por las tumbas del Sacerdote Lechuza y la Sacerdotisa. 0 tal vez -el tiempo y las excavaciones posteriores en el área lo determinarán- sea sólo un pequeño atisbo de todo un cementerio de élite correspondiente al Periodo Mochica Medio y asociado a su proximidad con la Huaca Chodoff.

Para terminar, hay que hacer hincapié en el concepto de ancestralidad pero desligándolo de la carga familiar y viéndolo más como sinónimo de referente cultural, social o religioso. Creo que, de alguna forma, estas ceremonias de entierro funcionaban como un obituario en el que, no sólo se recordaba y honraba la vida pasada del individuo enterrado, sino que se trataba de dejar un sello de su identidad hacia el futuro; de esta forma, sus acólitas, que bien podían ser el resto de mujeres de su séquito o las nuevas Sacerdotisas que fueran a heredar el cargo, podían reflejarse en ella como inspiración. En cierto modo, más allá de que la familia o la comunidad enterraran a un familiar o amigo, se trataba de recrear la pervivencia de su identidad. Así pues, el individuo moría pero el personaje pervivía para que la sociedad lo retomara con más fuerza mediante otro individuo que se encargaba de encarnar el papel de Sacerdote Lechuza o Sacerdotisa.

\section{Bibliografía}

ALVA, Walter, 2004. Sipán, Descubrimiento e Investigación. Lima, Perú

ALVA, Walter y Christopher B. DONNAN, 1993. Royal Tombs of Sipán. Fowler Museum of Cultural History, University of California, Los Angeles.

BOURGET, Steve, 2006. Death, Sex, and Sacrifice in Moche Religion and Visual Culture. Austin, University of Texas Press.

CASTILLO BUTTERS, Luis Jaime, 2000. Los rituales Mochicas de la muerte. En: Los Dioses del Antiguo Perú, Krzysztof Makowski y otros, págs. 103-135. Colección Arte y Tesoros del Perú. Lima, Banco de Crédito del Perú.

CASTILLO BUTTERS, Luis Jaime, 2003. Los Últimos Mochicas en Jequetepeque. En: Moche: Hacia el Final del Milenio, Actas del $2^{\circ}$ Coloquio de Cultura Moche (Trujillo, 1 al 7 de Agosto de 1999) Santiago Uceda y Elías Mujica, editores, T. II, págs. 65-123. Lima, Universidad Nacional de Trujillo and Pontificia Universidad Católica del Perú.

CASTILLO BUTTERS, Luis Jaime, 2005a. Las Señoras de San José de Moro, Rituales funerarios en la costa norte del Perú. En: Divina y Humana, La Mujer en los Antiguos México y Perú, Instituto Nacional de Antropología e Historia/Conaculta, México.

CASTILLO BUTTERS, Luis Jaime, 2005b. Gallinazo, Vicús y Moche en el desarrollo de las sociedades complejas de la costa norte del Perú. En: Actas del Primer Simposium sobre la Cultura Gallinazo, editado por Jean Francoise Millaire, págs. xxx-xxx. Cotsen Institute of Archaeology, Los Angeles.

CASTILLO BUTTERS, Luis Jaime (Editor), 2005-2017. Informes técnicos del Programa Arqueológico San José de Moro, Temporada 2004-2017, Edición Digital, Luis Jaime Castillo, Editor, Lima, Pontificia Universidad Católica del Perú.

CASTILLO BUTTERS, Luis Jaime y Hélène BERNIER, Gregory LOCKARD y Julio RUCABADO (Editores), 2008. Arqueología Mochica, Nuevos Enfoques, Actas de la Primera Conferencia Internacional de Jóvenes Investigadores de la Cultura Mochica. Fondo Editorial de la Pontificia Universidad Católica del Perú, Lima.

CASTILLO BUTTERS, Luis Jaime y Christopher B. DONNAN, 1994a. La ocupación Moche de San José de Moro, Jequetepeque. En: Moche: propuestas y perspectivas. Actas del Primer Coloquio sobre la Cultura Moche (Trujillo, 12 al 16 de abril de 1993), Santiago Uceda y Elías Mujica, editores. Travaux de l'Institute Français d'Etudes Andines 79: 93-146. Lima, Universidad de La Libertad - Trujillo, Instituto Francés de Estudios Andinos y Asociación Peruana para el Fomento de las Ciencias Sociales.

CASTILLO BUTTERS, Luis Jaime y Christopher B. DONNAN, 1994b. Los Mochicas del norte y los Mochicas del sur, una perspectiva desde el valle de Jequetepeque. En: Vicús, Krzysztof Makowski y otros, págs. 143-181. Colección Arte y Tesoros del Perú. Lima, Banco de Crédito del Perú. 
CASTILLO BUTTERS, Luis Jaime y Ulla HOLMQUIST PACHAS, 2000. Mujeres y poder en la sociedad Mochica tardía. En: El hechizo de las imágenes. Estatus social, género y etnicidad en la historia peruana, Narda Henríquez, compiladora, págs. 13-34. Lima, Fondo Editorial de la Pontificia Universidad Católica del Perú.

CASTILLO BUTTERS, Luis Jaime, Julio RUCABADO YONG, Martín DEL CARPIO PERLA, Katiuska BERNUY QUIROGA, Karim RUIZ ROSELL, Carlos RENGIFO CHUNGA, Gabriel PRIETO BURMESTER y Carole FRARESSO, 2008. Ideología y Poder en la Consolidación, Colapso y Reconstitución del Estado Mochica del Jequetepeque. El Proyecto Arqueológico San José de Moro (1991 - 2006). En: Ñawpa Paccha 26: Berkeley 2008, Institute of Andean Studies.

CASTILLO BUTTERS, Luis Jaime y Santiago UCEDA CASTILLO, 2008. The Mochicas. In: Handbook of South American Archaeology, editado por Helaine Silverman y William Isbell, Chapter 36:707-729. Springer, New York.

CASTILLO BUTTERS, Luis Jaime y Carlos RENGIFO, 2006. Arquitectura Funeraria en San José de Moro. Diseño arquitectónico de un Cementerio a inicios del segundo milenio. En: Programa Arqueológico San José de Moro, Temporada 2005. Pp. 8-43. Luis Jaime Castillo, editor. Pontificia Universidad Católica del Perú.

CASTILLO BUTTERS, Luis Jaime y Carlos RENGIFO 2008. The Funerary Identity of Specialists. The San Jose de Moro Cases and the Construction of the Identity during the Transitional Period. Actas del Simposio Modelos y Prácticas Funerarias Antiguos en América del Sur, 2-5 de abril de 2008, Lovaina, Bélgica.

Del CARPIO, Martín, 2008. La Ocupación Mochica Medio en San José de Moro. En: Arqueología Mochica: Nuevos Enfoques, Actas del Primer Congreso Internacional de Jóvenes Investigadores sobre la Cultura Mochica (Lima, 4 y 5 de Agosto del 2004). Págs. 81-104. Tomo 21 de la colección „Actes \& Mémories de l'Institut Français d'Études Andines“. Luis Jaime Castillo, Julio Rucabado, Hélène Bernier y Gregory Lockard, editores. Fondo Editorial de la Pontifica Universidad Católica del Perú e Instituto Francés de Estudios Andinos.

DISSELHOFF, Hans Dietrich, 1958. Tumbas de san José de Moro (Provincia de Pacasmayo, Perú). En Proceedings of the 32nd International Congres of Americanists (Copenhagen, 1956), pp. 364-367. Copenhagen.

DONNAN, Christopher B., 1995. Moche funerary practice. En: Tombs for the Living: Andean Mortuary Practices, editado por T. D. Dillehay, pp. 111-159. Dumbarton Oaks Research Library and Collection, Washington, D.C.

DONNAN, Christopher B., 2008. Moche Tombs at Dos Cabezas, Cotsen Institute of Archaeology (UCLA), Los Angeles.

DONNAN, Christopher B. y Luis Jaime CASTILLO, 1992. Finding the tomb of a Moche priestess. Archaeology 6 (45): 3842. New York, Archaeological Institute of America.
DONNAN, Christopher B. y Luis Jaime CASTILLO, 1994. Excavaciones de tumbas de sacerdotisas Moche en San José de Moro, Jequetepeque. En: Moche: propuestas y perspectivas. Actas del 1er Coloquio sobre la Cultura Moche (Trujillo, 12 al 16 de abril de 1993), Santiago Uceda y Elías Mujica, editores. Travaux de l'Institute Français d'Etudes Andines 79: 415-424. Lima, Universidad La Libertad-Trujillo, Instituto Francés de Estudios Andinos y Asociación Peruana para el Fomento de las Ciencias Sociales.

DONNAN, Christopher B, y Cock, Guillermo A., 1986. Pacatnamu Papers. Vol. I, The Cotsen Institute of Archaeology Press (UCLA), Los Angeles.

DONNAN, Christopher B. y Carole MACKEY, 1978. Ancient Burial patterns of the Moche Valley, Peru, University of Texas Press. Austin, USA.

DONNAN, Christopher B. y McCLELLAND, Donna, 1999. Moche Fineline Painting: Its Evolution and Its Artists. Fowler Museum of Cultural History, University of California Los Angeles, Los Angeles.

FRARESSO, Carole, 2007. L'usage du métal dans la parure el les rites de la culture Mochica (150-850 AP. J-C), Pérou. Tesis Doctoral presentada en L'Université Michel De Montaigne Bordeaux 3.

GOEPFERT, Nicolas, 2006. El contexto de camélidos A38. En: Programa Arqueológico San José de Moro, Temporada 2006. Luis Jaime Castillo, editor. Págs. 70-162. Pontificia Universidad Católica del Perú.

McCLELLAND, Donna D., Don McCLELLAND y Christopher B. DONNAN, 2007. Moche Fineline Painting from San José de Moro. The Cotsen Institute of Archaeology at University of California, Los Angeles.

MAKOWSKI HANULA, Krzysztof, 2000. Las divinidades en la iconografía Mochica. En: Los dioses del antiguo Perú, Krzysztof Makowski y otros, págs. 137-175. Colección Arte y Tesoros del Perú. Lima, Banco de Crédito del Perú.

QUILTER, Jeffrey, 2002. Moche Politics, Religion and Warfare. En: Journal of World Prehistory, 16(2): 145-195. Plenum Publishing Corporation.

RENGIFO, Carlos y Alfonso BARRAGÁN, 2005. Excavaciones en el Área 33 de San José de Moro. En: Programa Arqueológico San José de Moro, Temporada de excavaciones 2004. Luis Jaime Castillo, editor. Págs. 114-164. Informe Técnico presentado al Instituto Nacional de Cultura, Lima.

RENGIFO, Carlos; Daniela ZEVALLOS y Luis MURO, 2007. Excavaciones en las áreas 28, 33, 34, 40 y 43. La ocupación Mochica en el sector norte de San José de Moro. En: Programa Arqueológico San José de Moro, Temporada 2007. Luis Jaime Castillo, editor. Págs. 162-209. Pontificia Universidad Católica del Perú.

RUIZ ROSELL, Karim, Rauolas Cecil, Rucabado Julio y Barrazueta Roxana, 2005. Excavaciones en las área 38. En: Programa Arqueológico San José de Moro, Temporada 2005. Luis Jaime Castillo, editor. Págs. 70-162. Pontificia Universidad Católica del Perú. 
RUIZ ROSELL, Karim, Rucabado Julio y Barrazueta Roxana, 2006. Excavaciones en las área 38. En: Programa Arqueológico San José de Moro, Temporada 2006. Luis Jaime Castillo, editor. Págs. 70-162. Pontificia Universidad Católica del Perú.

RUIZ ROSELL, Karim, Rucabado Julio y Barrazueta Roxana, 2007. Excavaciones en las área 38. En: Programa Arqueológico San José de Moro, Temporada 2007. Luis Jaime Castillo, editor. Págs. 70-162. Pontificia Universidad Católica del Perú.

RUIZ ROSELL, Karim, 2008. La tumba M-U1411: un entierro Mochica medio de élite en el cementerio de San José de Moro. En Arqueología Mochica, Nuevos Enfoques, Págs.
381-396. Actas de la Primera Conferencia Internacional de Jóvenes Investigadores de la Cultura Mochica. Fondo Editorial de la Pontificia Universidad Católica del Perú, Lima. RUIZ ROSELL, Karim, 2013. Oficiantes Mochica Medio en San José de Moro: el "Sacerdote lechuza" y la "Sacerdotisa", Tesis Doctoral, Universitat Autònoma de Barcelona, Barcelona.

SCHUlEnBERG, Thomas S., 2010. Aves de Perú, Centro de Ornitología y Biodiversidad, 2010, Lima.

UBBELOHDE-DOERING, Heinrich, 1983. Vorspanische Gräber von Pacatnamú, Nordperu, Materialien zur Allgemeinen und Vergleichenden Archäologie, Band 26, 1983, München. 